\title{
Inhibition of Class I Histone Deacetylases Abrogates Tumor Growth Factor $\beta$ Expression and Development of Fibrosis during Chronic Pancreatitis $\mathbf{s}$
}

\author{
Marta Bombardo, Rong Chen, Ermanno Malagola, Enrica Saponara, Andrew P. Hills, \\ Rolf Graf, and Sabrina Sonda ${ }^{1}$
}

Swiss Hepato-Pancreato-Biliary Center, Department of Visceral and Transplantation Surgery, University Hospital (M.B., R.C., E.M., E.S., R.G., S.S.) and Center for Integrative Human Physiology (ZIHP), University of Zurich (R.G., S.S.), Zurich, Switzerland; and School of Health Sciences, College of Health and Medicine, University of Tasmania, Launceston, Australia (A.P.H., S.S.)

Received October 23, 2017; accepted May 31, 2018

\section{ABSTRACT}

Pancreatic fibrosis is the hallmark of chronic pancreatitis, a highly debilitating disease for which there is currently no cure. The key event at the basis of pancreatic fibrosis is the deposition of extracellular matrix proteins by activated pancreatic stellate cells (PSCs). Transforming growth factor $\beta$ (TGF $\beta$ ) is a potent profibrotic factor in the pancreas as it promotes the activation of PSC; thus, pharmacologic interventions that effectively reduce TGF $\beta$ expression harbor considerable therapeutic potential in the treatment of chronic pancreatitis. In this study, we investigated whether TGF $\beta$ expression is reduced by pharmacologic inhibition of the epigenetic modifiers histone deacetylases (HDACs). To address this aim, chronic pancreatitis was induced in C57BL/6 mice with serial injections of cerulein, and the selective class 1 HDAC inhibitor MS-275 was administered in vivo in a preventive and therapeutic manner. Both MS-275 regimens potently reduced deposition of extracellular matrix and development of fibrosis in the pancreas after 4 weeks of chronic pancreatitis. Reduced pancreatic fibrosis was concomitant with lower expression of pancreatic TGF $\beta$ and consequent reduced PSC activation. In search of the cell types targeted by the inhibitor, we found that MS-275 treatment abrogated the expression of TGF $\beta$ in acinar cells stimulated by cerulein treatment. Our study demonstrates that MS-275 is an effective antifibrotic agent in the context of experimental chronic pancreatitis and thus may constitute a valid therapeutic intervention for this severe disease.

\section{Introduction}

Chronic pancreatitis is defined as a progressive inflammation of the pancreas, resulting in development of organ fibrosis, which is at the core of the disease pathophysiology. This progressive condition is characterized by irregular sclerosis with focal, segmental, or diffuse destruction of the parenchyma. Consequently, gradual loss of exocrine and endocrine cellular components leads to pancreatic insufficiency and eventually diabetes, which are associated with considerable morbidity, reduction of quality of life, and reduction of life expectancy (reviewed in DiMagno and DiMagno, 2013, 2016).

Fibrosis is characterized by excessive production and deposition of extracellular matrix (ECM) components in the pancreatic parenchyma, produced mainly by resident pancreatic stellate cells (PSCs). In response to organ injury,

This work received funding from the Swiss National Science Foundation (grant no. 310030 -146725) and the Amélie Waring Foundation.

${ }^{1}$ Current affiliation: School of Health Sciences, College of Health and Medicine, University of Tasmania, Newnham Campus, Launceston, Australia https://doi.org/10.1124/mol.117.110924.

S This article has supplemental material available at molpharm. aspetjournals.org. profibrogenic factors are released and activate PSCs, a process characterized by phenotypical cell alteration, proliferation, and ECM protein synthesis.

Despite advances in chronic pancreatitis research, to date, the complex cellular and signaling mechanisms that drive the fibrotic process are not yet completely elucidated. This limited knowledge explains why therapeutic approaches to counteract the development of organ fibrosis are not currently available and management of chronic pancreatitis remains a clinical challenge.

In this study, we evaluated whether administration of MS-275 (also known as entinostat), a selective inhibitor of class 1 histone deacetylases (HDACs), counteracts the development of pancreatic fibrosis using the widespread murine model of cerulein-induced chronic pancreatitis. The rationale for this approach was 3-fold: 1) development of fibrosis activates a substantial gene regulation, which is prominently orchestrated by epigenetic mechanisms (McDonnell et al., 2014; Weigel et al., 2015; Yang and Schwartz, 2015; MoranSalvador and Mann, 2017); 2) HDACs are critical epigenetic regulators, and expression of class 1 HDACs is significantly upregulated during the course of chronic pancreatitis (Bombardo et al., 2017); and 3) pharmacologic inhibitors of 
HDAC activity, originally developed as anticancer agents, are currently being investigated for their antifibrotic properties in different fibrotic diseases (recently reviewed in Pang and Zhuang, 2010; Royce et al., 2014; Chen et al., 2015; Schuetze et al., 2016).

\section{Materials and Methods}

Animal Experiments. All animal treatments were performed in accordance with Swiss federal animal regulations and approved by the cantonal veterinary office of Zurich. All studies involving animals were carried out in accordance with the Guide for the Care and Use of Laboratory Animals as adopted and promulgated by the U.S. National Institutes of Health. Mice used in this study were adult 8- to 10-weekold wild-type C57BL/6 mice in a weight range of 25-30 g (Envigo Laboratories, Horst, The Netherlands). Animals were kept under standardized conditions under 12-hour light/dark cycles, with food and water available ad libitum. Groups of 4 to 5 mice were kept in standard individually ventilated cages in a specific pathogen-free facility. Food and water were provided ad libitum. Only male mice were used in this study.

Chronic pancreatitis was induced via six intraperitoneal injections of cerulein $(50 \mu \mathrm{g} / \mathrm{kg})$ administered hourly every 2 nd day for up to 6 weeks. Control animals received $0.9 \% \mathrm{NaCl}$ injections. MS-275 (Selleckchem, Houston, TX) was injected intraperitoneally at $20 \mathrm{mg} / \mathrm{kg}$ every 2nd day for 2 weeks, starting concomitantly (preventive regimen) or 1 week after the beginning of cerulein injections (therapeutic regimen). The concentration of MS-275 was chosen based on previously published in vivo studies using the inhibitor in mice (Dalgard et al., 2008; Nguyên et al., 2008; Murphy et al., 2014; Bombardo et al., 2017). Control animals received $10 \%$ dimethylsulfoxide injections. Intraperitoneal injections were alternated daily between the left and right sides of the abdomen. Mice were examined throughout the development of pancreatitis, and their health status was recorded every 2nd day on a score sheet. Pancreatitis models used in this study generated only a mild form of the disease. Animal weight loss did not exceed $10 \%$, and no mortality was observed. After deep terminal anesthesia with isoflurane, mice were euthanized via cardiac puncture exsanguination. Groups of five animals were tested for each experiment. Animals were assigned randomly to different experimental groups for all in vivo studies. Data collection and evaluation of all in vivo and in vitro experiments were performed blinded to group identity.

Mammalian Cell Cultures. Cell culture reagents were from Gibco-BRL. Rat AR42J cells were maintained in Kaighn's modified Ham's F-12 medium with $20 \%$ fetal bovine serum, supplemented with $50 \mathrm{U} / \mathrm{ml}$ penicillin and $50 \mu \mathrm{g} / \mathrm{ml}$ streptomycin, and maintained at stable condition of $37^{\circ} \mathrm{C}$ in a $5 \% \mathrm{CO}_{2}$ atmosphere. Cells were seeded in six-well plates, stimulated with $10 \mathrm{nM}$ cerulein for 4 hours, and lysed in the plates for RNA extraction and real-time polymerase chain reaction (PCR) analysis.

Primary acini were isolated according to Algül et al. (2007) from 6-week-old Wistar male rats from Charles River, Deutschland. Acini were preincubated with $1 \mu \mathrm{M}$ MS-275 for 30 minutes and stimulated with $0.1 \mathrm{nM}$ cerulein for 30 minutes in the presence of $1 \mu \mathrm{M}$ MS-275. At the end of the treatment, cells were lysed in the plates for RNA extraction and real-time PCR analysis.

Immunohistochemistry. Pancreas specimens were embedded in paraffin for histologic analyses, as previously described (Silva et al., 2011). H\&E and Masson's trichrome staining were performed according to routine procedures. Microscopy analyses were performed on a wide-field Nikon Eclipse Ti (Amsterdam, The Netherlands). Quantification of labeled cells was performed in at least 10 randomly selected high-power fields $(\times 200)$ per slide using the NIS Elements BR Analysis (Nikon, Amsterdam, The Netherlands) and Cell^ $\mathrm{P}$ analysis software (Olympus, Tokyo, Japan).
Western Blotting. Twenty milligrams of pancreatic tissue was homogenized in radioimmunopreciptation (RIPA) assay buffer containing a protease inhibitor cocktail (Roche Diagnostics, Mannheim, Germany). Protein concentrations were determined by a Bradford protein assay (BioRad, Hercules, CA). Twenty micrograms of proteins was resolved by SDS-PAGE electrophoresis and blotted onto nitrocellulose membranes using a V3 Western Workflow system (BioRad) according to the manufacturer's protocols.

Membranes were incubated with primary antibodies overnight at $4^{\circ} \mathrm{C}$. Primary antibodies used in this study were: mouse anti- $\alpha$-smooth muscle actin ( $\alpha$-SMA, Dako, Glostrup, Denmark); rabbit anti $\alpha$-tubulin (ab52894; Abcam, Cambridge, UK); rabbit anti-phospho-Smad3 (Ser423/425) (Cell Signaling, Danvers, MA); and rabbit anti-GAPDH (Santa Cruz Biotechnology, Dallas, TX).

Nuclear Protein Extraction and HDAC Activity. Nuclear proteins were extracted from $20 \mathrm{mg}$ of pancreatic tissue with the EpiQuik Nuclear Extraction Kit (Epigentek Group Inc, Mountain View, CA), and HDAC activity was measured in the nuclear extracts with the fluorimetric EpiQuik HDAC activity/inhibition assay kit (Epigentek Group Inc.), according to the manufacturer's instructions.

Transcript Analyses. Total RNA was extracted from pancreatic tissue and acinar explants as described previously (Graf et al., 2002) and reverse-transcribed with qScript cDNA SuperMix (Quanta Biosciences, Beverly, MA). Gene expression was measured by real-time PCR on a 7500 Fast Real-Time PCR System (Applied Biosystems, Carlsbad, CA) using Taqman probes (Applied Biosystems). Transcript levels were normalized using $18 \mathrm{~S}$ RNA as a reference and expressed as $\Delta \Delta \mathrm{Ct}$ relative to the value of control animals or as $\Delta \mathrm{Ct}$.

Statistical Analyses. Every group of mice compared in the different experimental conditions was comprised 5 animals. Data are expressed as means \pm standard deviation. Population characteristics were compared among treatment groups using an unpaired, twotailed Student's $t$ test when comparing two experimental conditions or one-way analysis of variance, followed by Dunnett's post-hoc test when comparing more than two experimental conditions. HolmBonferroni correction for multiple comparisons was used to keep the family-wise error rate of dependent variables at 5\%. Analyses were performed using GraphPad Prism 4.0c (GraphPad Software, Inc., San Diego, CA).

\section{Results}

Development of Fibrotic Response during Chronic Pancreatitis Correlates with Increased Levels of HDAC Expression. To investigate the role of HDAC in the development of pancreatic fibrosis after induction of chronic pancreatitis, we first performed a time-course analysis after induction of the disease to determine the kinetics of the fibrotic response. Histologic evaluation of mice harvested after 2, 4, and 6 weeks of cerulein treatment revealed progressive damage of pancreatic parenchyma and cell infiltration (Fig. 1A) and pronounced ECM deposition (Fig. 1B). Quantification of fibrotic parameters showed increased expression of collagen isoforms (Fig. 1C) and collagen deposition in the pancreas (Fig. 1D). Development of pancreatic fibrosis is mediated by activated pancreatic stellate cells (PSCs), which constitute the predominant source of ECM proteins, including collagens and fibronectin. Activation of PSCs, detected by $\alpha$-smooth muscle actin ( $\alpha$-SMA) expression, reached a maximum after 4 weeks of pancreatitis (Fig. 1E), thus mirroring the kinetics of collagen expression. Expression of profibrotic $\mathrm{TGF} \beta$ isoforms, the main activators of PSC, and TGF $\beta$ receptor II, critical for the development of pancreatic fibrosis (Yoo et al., 2005), also increased in a similar pattern during 
A

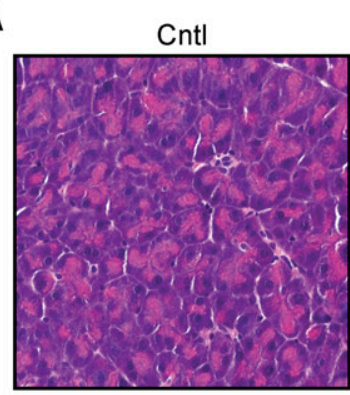

B

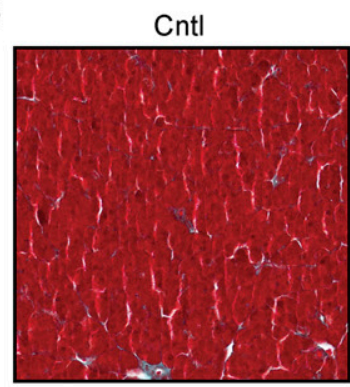

H\&E
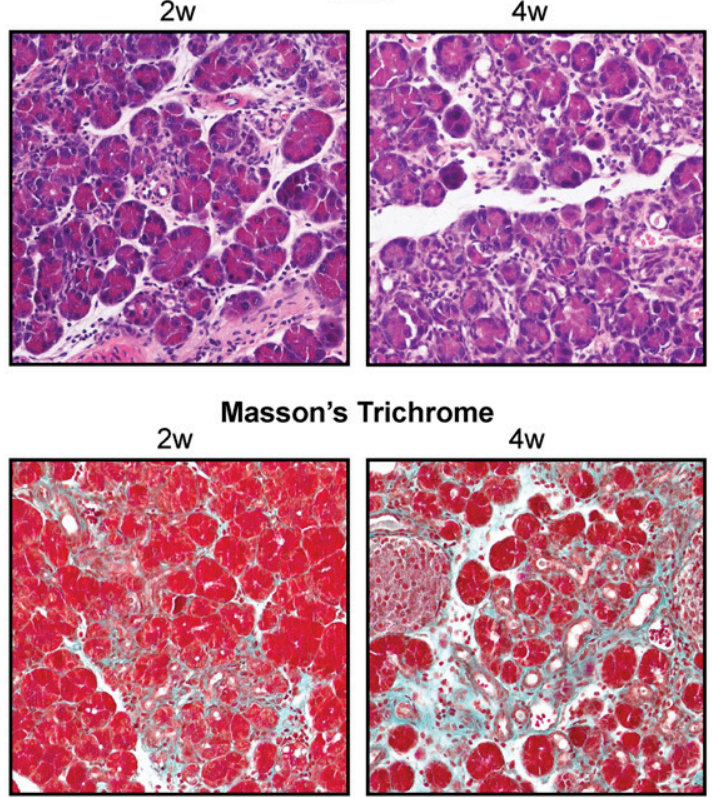

$4 w$

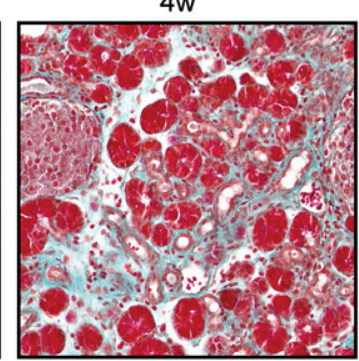

$6 w$

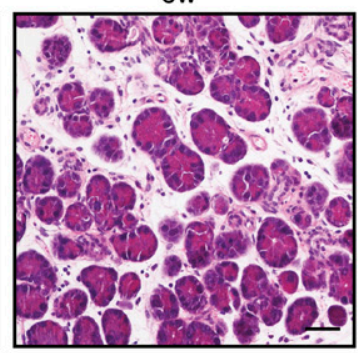

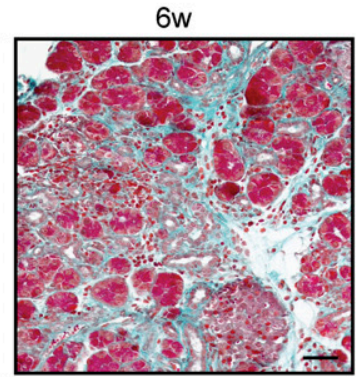

C
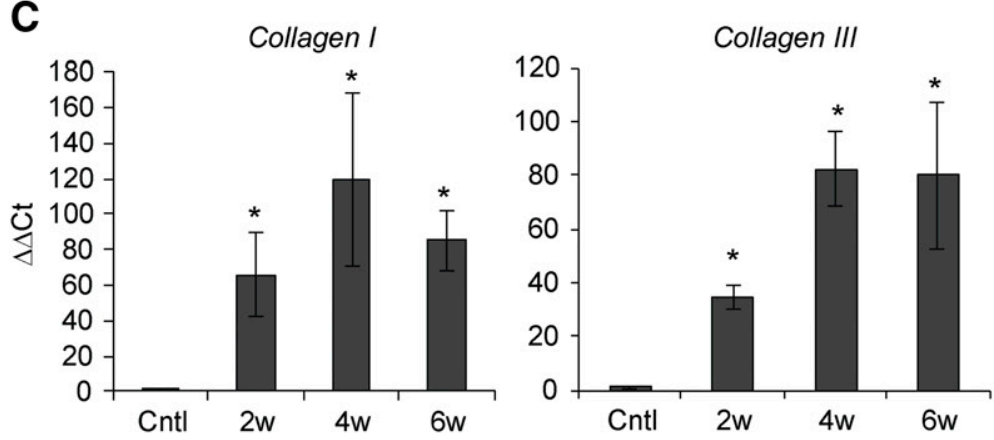

D

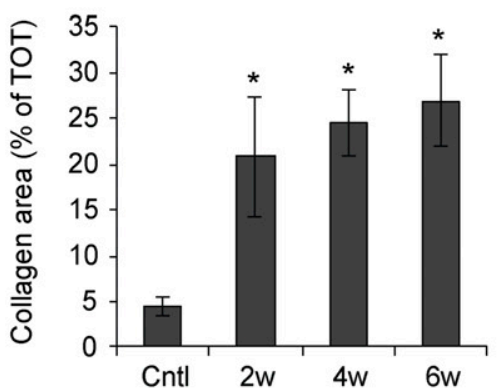

E

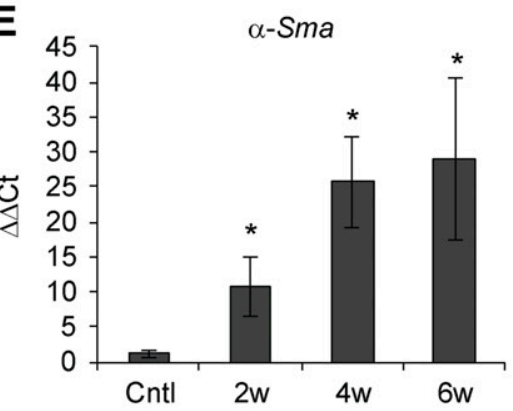

$\mathbf{F}$

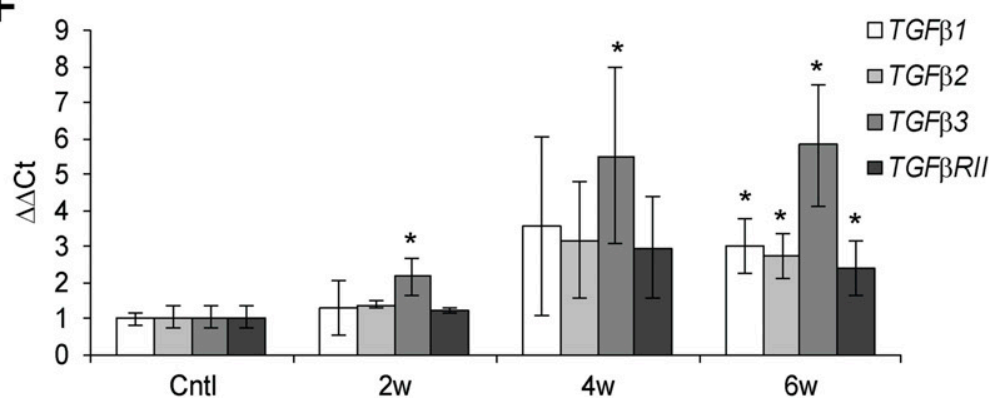

Fig. 1. Characterization of chronic pancreatitis development. (A) H\&E and (B) Masson's trichrome staining of pancreata at the indicated weeks of pancreatitis induction revealed progressive destruction of parenchymal morphology, cell infiltration, and collagen deposition (green staining). (C) Quantitative PCR (qPCR) of collagen 1 and 3 expression in the pancreas at the indicated weeks of pancreatitis. (D) Quantification of collagen deposition, based on Masson's trichrome staining, at the indicated weeks of pancreatitis. The amount of collagen is expressed as the percentage of total pancreatic area. (E) qPCR of $\alpha$-SMA expression in the pancreas at the indicated weeks of pancreatitis, indicative of pancreatic stellate cell activation. (F) qPCR of $\mathrm{TGF} \beta$ isoforms and TGF $\beta$ receptor 2 (TGF $\beta$ R2) expression in the pancreas at the indicated weeks of pancreatitis. Results are average \pm S.D. $(n=5), * P<$ 0.05. Scale bar, $50 \mu \mathrm{m}$.

the development of organ fibrosis (Fig. 1F). In addition, pancreatic expression of inflammatory components followed similar kinetics (Supplemental Fig. 1) (Bombardo et al., 2017). Based on these results showing maximal levels of fibrosis after 4 weeks of pancreatitis, we focused on this time point for further analyses.
Inhibition of Class 1 HDAC with MS-275 Reduces the Development of Fibrosis after Induction of Chronic Pancreatitis. We recently showed that gene expression levels of class 1 HDACs were upregulated during chronic pancreatitis (Bombardo et al., 2017). This finding was confirmed by increased HDAC enzymatic activity (Fig. 2A) in 


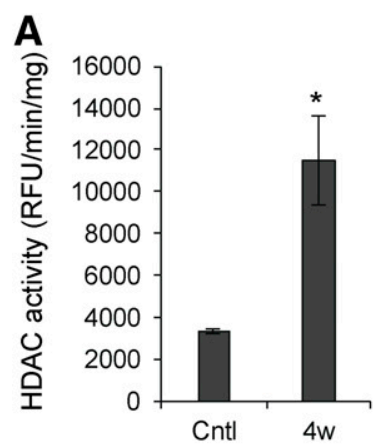

\begin{tabular}{|c|c|c|c|c|}
\hline Time & 1 week & 2 weeks & 3 weeks & 4 weeks \\
\hline Cntl & $\mathrm{NaCl}$ & $\mathrm{NaCl}$ & $\mathrm{NaCl}$ & $\mathrm{NaCl}$ \\
\hline MS & $\begin{array}{l}\mathrm{MS} \\
\mathrm{NaCl}\end{array}$ & $\begin{array}{l}\mathrm{MS} \\
\mathrm{NaCl}\end{array}$ & $\mathrm{NaCl}$ & $\mathrm{NaCl}$ \\
\hline Cer & Cerulein & Cerulein & Cerulein & Cerulein \\
\hline $\begin{array}{l}\text { Preventive } \\
\text { (MS+Cer) }\end{array}$ & $\begin{array}{c}\text { MS } \\
\text { Cerulein }\end{array}$ & $\begin{array}{c}\text { MS } \\
\text { Cerulein }\end{array}$ & Cerulein & Cerulein \\
\hline $\begin{array}{l}\text { Therapeutic } \\
\text { (Cer+MS) }\end{array}$ & Cerulein & $\begin{array}{c}\text { MS } \\
\text { Cerulein }\end{array}$ & $\begin{array}{c}\text { MS } \\
\text { Cerulein }\end{array}$ & Cerulein \\
\hline
\end{tabular}
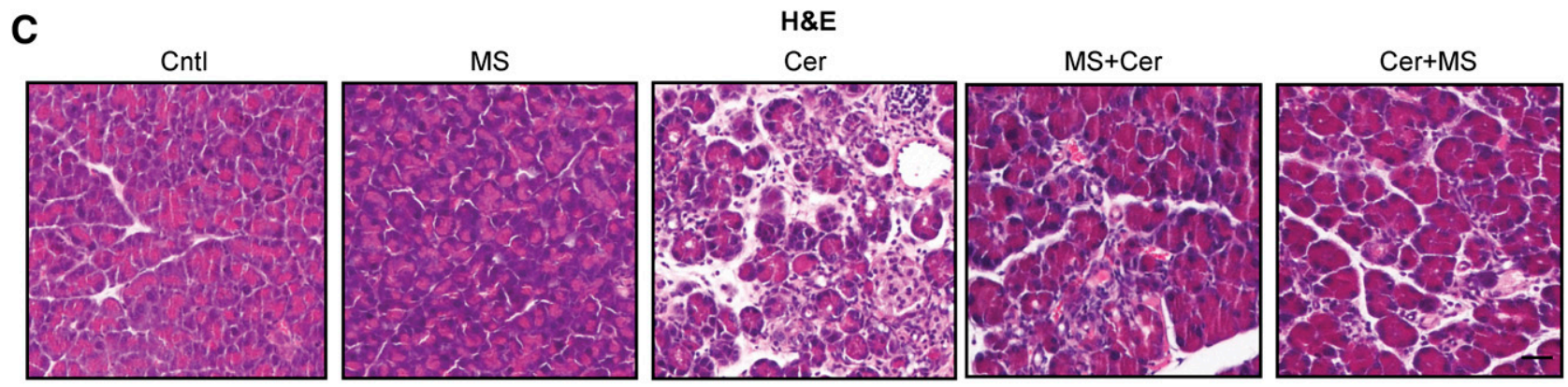

D
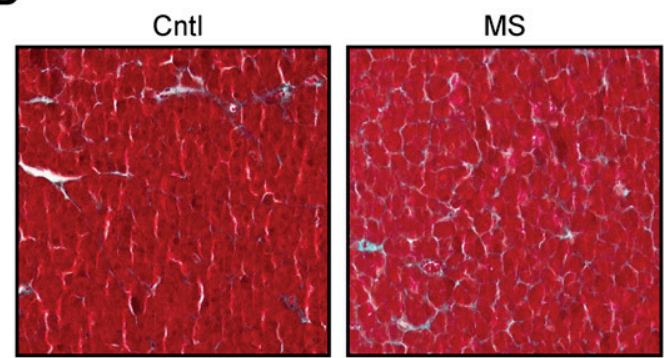

\section{Masson's Trichrome}
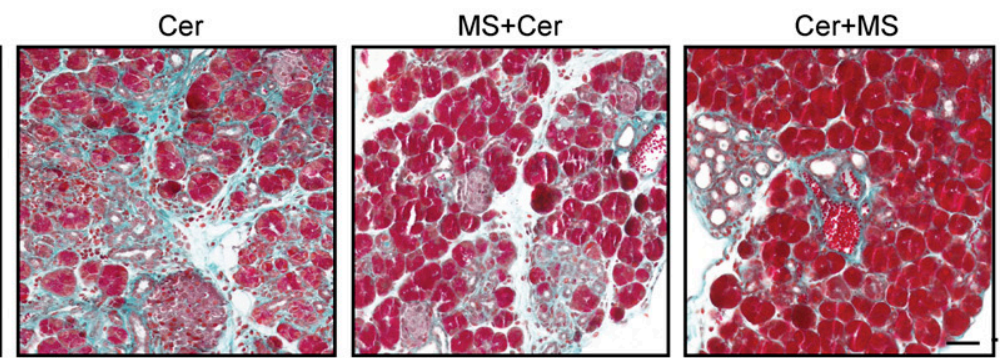

E
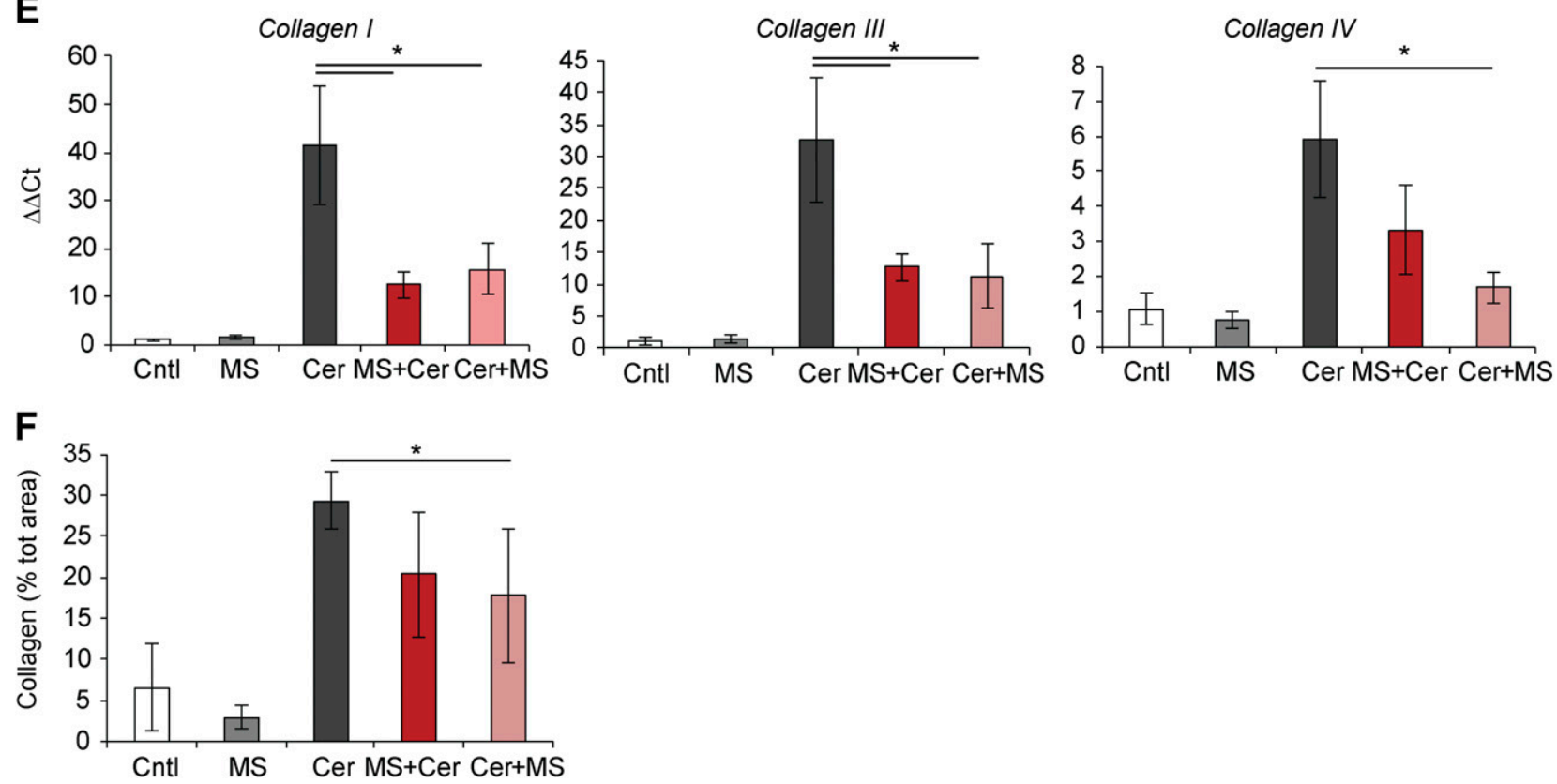

Fig. 2. Preventive and therapeutic administration of MS-275 reduces the development of fibrosis during chronic pancreatitis. (A) Total HDAC activity detected in pancreatic nuclear extract in control and mice treated with cerulein (Cer) for 4 weeks mice. (B) Schematic representation of 2 weeks of preventive (MS + Cer) and therapeutic (Cer + MS) MS-275 regimens during induction of chronic pancreatitis. Cer was administered on alternate days over 4 weeks. MS-275 was administered on alternate days over 2 weeks. (C) H\&E staining of pancreata after 4 weeks of chronic pancreatitis after preventive and therapeutic MS-275 regimens. (D) Masson's trichrome staining of pancreata showing reduced collagen deposition (green) after 4 weeks of 
pancreatic nuclear proteins after 4 weeks of cerulein treatment. To test whether class 1 HDAC upregulation was functionally linked to the development of fibrosis, we treated mice with the selective class 1 inhibitor MS-275, which we previously showed to potently inhibit HDAC activity in the pancreas (Bombardo et al., 2017). The inhibitor was administered for 2 weeks in a preventive manner, starting concomitantly with the first cerulein injection. Alternatively, we administered the inhibitor in a therapeutic manner, starting 1 week after the induction of pancreatitis. Animals were harvested after 4 weeks of chronic pancreatitis according to the regimens depicted in Fig. 2B. Both modalities of MS-275 treatment resulted in a trend of reduced expression of selected HDAC isoforms (Supplemental Fig. 2) and better preservation of pancreatic parenchyma (Fig. 2, C and D). This result was further confirmed by reduced expression of collagen isoforms (Fig. 2E) and lower collagen deposition (Fig. 2F) after MS-275 administration. As collagen is deposited mainly by activated PSCs, we next evaluated whether PSC activation was limited in the presence of MS-275. Expression of $\alpha$-SMA, a key hallmark of PSC activation, was lower upon MS-275 treatment at both RNA (Fig. 3A) and protein levels (Fig. 3B). These data revealed that both preventive and therapeutic regimens of MS-275 were effective in reducing PSC activation and consequently limiting the development of fibrotic processes on induction of chronic pancreatitis.

Inhibition of Class 1 HDAC with MS-275 Reduces TGF $\beta$ Expression after Induction of Chronic Pancreatitis. Activation of PSCs is initiated by damaged acinar cells and reinforced by already activated PSCs in a paracrine and autocrine manner through the synthesis and secretion of profibrotic mediators (Apte et al., 2011). Thus, we investigated whether the reduced fibrosis observed upon MS-275 treatment was linked to reduced expression of these factors. In support of this hypothesis, gene expression levels of profibrotic TGF $\beta 1-3$ were lower in the presence of the inhibitor (Fig. 4A). Consequent to the decreased production of TGF $\beta$ isoforms, activation of TGF $\beta$ signaling was attenuated, as shown by a trend of reduced C-terminal phosphorylation that is required for activation of Smad3, an initiating event of the intracellular cascade resulting upon engagement and dimerization of $\mathrm{TGF} \beta$ receptor complex (Fig. 4B). Interestingly, TGF $\beta$ receptor 2 was also upregulated following induction of pancreatitis; however, its expression levels were not reduced after MS-275 treatment (Fig. 4C).

Inhibition of Class 1 HDAC with MS-275 Reduces TGF $\beta$ Expression in Pancreatic Acinar Cells. Previous works have shown that TGF $\beta$ is synthesized in activated PSC, thus acting through autocrine loops (reviewed in Apte et al., 2011); however, in our study, we investigated whether the synthesis of TGF $\beta$ upstream of PSC activation is inhibited by MS-275. Specifically, we asked whether: 1) acinar cells upregulate TGF $\beta$ expression after cerulein-induced pancreatitis and 2) MS-275 selectively interferes with this gene expression. In support of this hypothesis, we detected early upregulation of TGF $\beta 1$ in the pancreas 24 hours after cerulein
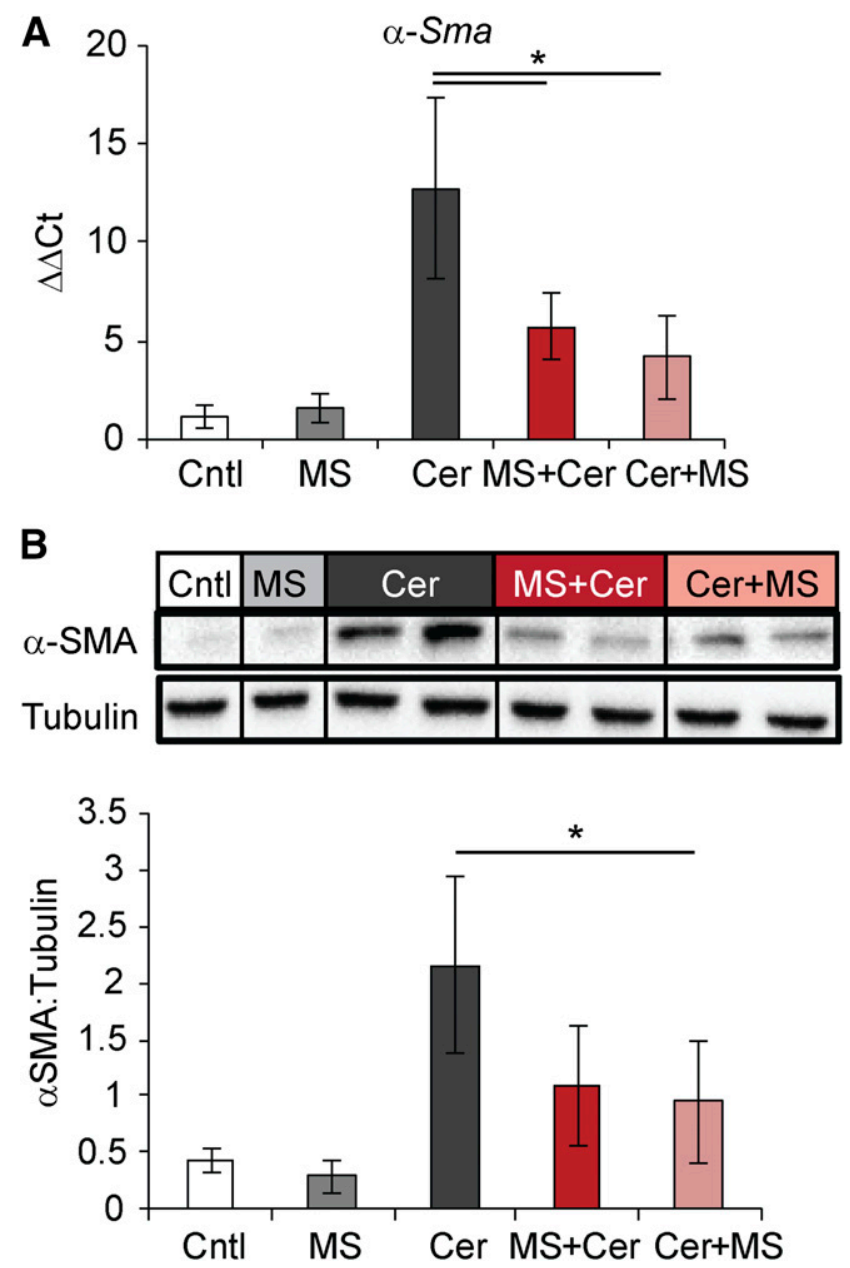

Fig. 3. Preventive and therapeutic administration of MS-275 reduces the activation of pancreatic stellate cells during chronic pancreatitis. (A) qPCR of $\alpha$-SMA expression in pancreata after 4 weeks of chronic pancreatitis after preventive and therapeutic MS-275 regimens. (B) Western blot quantification of $\alpha$-SMA in pancreata after 4 weeks of chronic pancreatitis after preventive and therapeutic MS-275 regimens. Band intensity values were normalized using tubulin as a loading control. Results are average \pm S.D. $(n=5),{ }^{*} P<0.05$.

treatment (Fig. 5A). Furthermore, in a new set of experiments, upregulation of TGF $\beta 1-2$ isoforms and TGF $\beta$ receptor 2 was observed in primary acini isolated 24 hours after cerulein treatment in vivo (Fig. $5 \mathrm{~B}$ ), suggesting that acini respond to the initial injury by upregulating TGF $\beta$ signaling components. We then tested whether TGF $\beta$ upregulation in acinar cells was a direct effect of cerulein administration or rather stimulated by stromal cells. AR42J acinar cells treated in vitro with cerulein upregulated TGF $\beta 1$ levels (Fig. 5C). Similarly, upregulation of TGF $\beta 1$ was observed when primary pancreatic acini were isolated and stimulated in vitro with cerulein (Fig. 5D). These data suggest that cerulein induced TGF $\beta 1$ gene expression in a cell-autonomous manner independent from the presence of stromal cells. Importantly, pretreatment

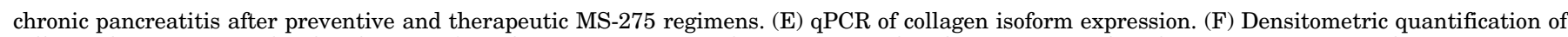

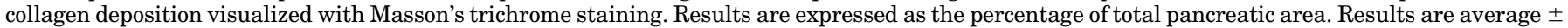
S.D. $(n=5),{ }^{*} P<0.05$. Scale bar, $50 \mu \mathrm{m}$. 

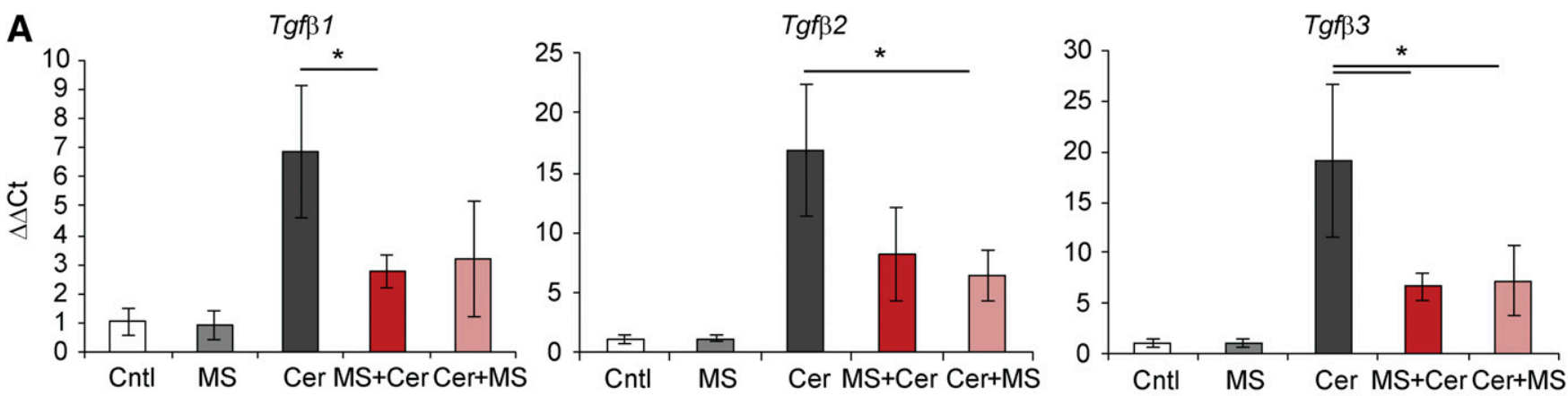

B
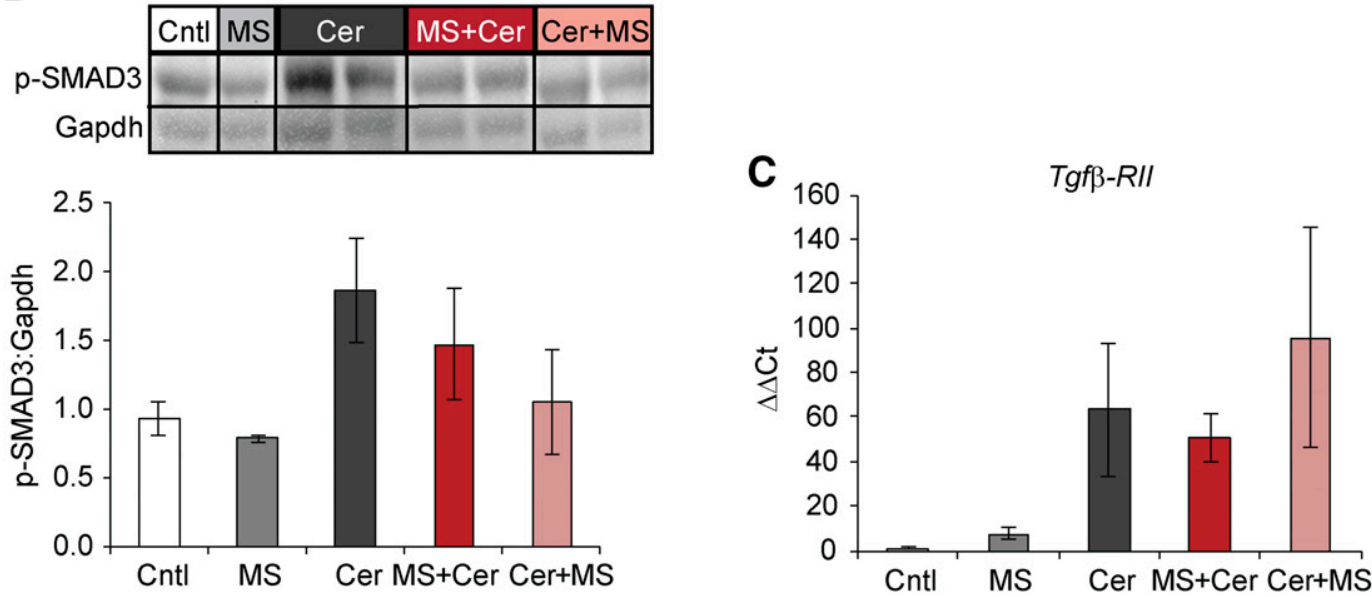

Fig. 4. Preventive and therapeutic administration of MS-275 reduces the expression of TGF $\beta$ during chronic pancreatitis. (A) qPCR of TGF $\beta$ isoforms expression after 4 weeks of chronic pancreatitis after preventive and therapeutic MS-275 regimens. (B) Western blotting quantification of phosphoSMAD3 in pancreata after 4 weeks of chronic pancreatitis after preventive and therapeutic MS-275 regimens. Band intensity values were normalized using Gapdh as a loading control (Cntl). (C) qPCR of TGF $\beta$ receptor 2 (TGF $\beta$ R2) expression in pancreata after 4 weeks of chronic pancreatitis after preventive and therapeutic MS-275 regimens. Results are average \pm S.D. $(n=5),{ }^{*} P<0.05$.

with the HDAC inhibitor MS-275 abrogated TGF $\beta 1$ induction in both AR42J cells and primary acini (Fig. 5, C and D). Furthermore, cerulein treatment increased expression of and HDAC1, but not HDAC2 and 3, in isolated acini (Fig. 5E), further suggesting that TGF $\beta 1$ expression in acinar cells is promoted by HDAC activity. Expression of TGF $\beta 2$ was much lower than TGF $\beta 1$ and not regulated in this experimental setting in both cell types (Supplemental Fig. 3, A and B).

\section{Discussion}

TGF $\beta$ is a potent fibrogenic factor that plays a pivotal role in the development of fibrosis during chronic pancreatitis (Menke et al., 1997; Yoo et al., 2005; He et al., 2009; Li et al., 2016). One of the main effects exerted by TGF $\beta$ is activation of PSCs from a quiescent state to a myofibroblast-like phenotype (reviewed in Apte et al., 2011). In recent years, activated PSCs have attracted increasing attention as major mediators of pancreatic fibrosis during chronic pancreatitis as they not only mediate the development of fibrosis by producing ECM proteins, but they also amplify the fibrotic response in an autocrine and paracrine manner by secreting fibrogenic factors, including TGF $\beta$ (Kruse et al., 2000). In the present study, we discovered that activity of HDACs in the pancreas is functionally linked to the development of fibrosis during chronic pancreatitis, thus providing a potential therapeutic target to counteract this disease. This hypothesis was further tested in in vivo experiments where the selective inhibitor of class 1 HDACs MS-275 was administered in a preventive or therapeutic manner during chronic pancreatitis. In both regimen types, we observed a striking inhibition of pancreatic fibrosis and increased preservation of pancreatic parenchyma, suggesting that MS-275 exerts an antifibrotic effect, even when administered after commencement of the disease.

At the cellular level, reduced fibrosis detected upon MS-275 treatment was likely the result of reduced TGF $\beta$ expression, leading to reduced activation of PSCs. An important question arising from these data is the identity of the cells whose TGF $\beta$ production is targeted by the inhibitor. Using in vitro experiments with isolated acinar cells, we found that short-term incubation with cerulein was sufficient to stimulate TGF $\beta$ expression in these cells. The fact that MS-275 treatment potently reduced cerulein-stimulated TGF $\beta$ expression suggests that acinar cells are indeed a direct target of the inhibitor and contribute to the phenotype observed in vivo. In this regard, it would be important to explore further the temporal regulation of TGF $\beta$ isoform expression in acinar cells to dissect the dynamic of their contribution to the development of pancreatic fibrosis.

It is known that acinar cells are not the only source of TGF $\beta$ in the pancreas, as previous studies reported the presence of TGF $\beta 1$ mRNA in stromal cells, including PSC, upon induction of pancreatitis (Muller-Pillasch et al., 1999). In this regard, it is important to mention that $\mathrm{TGF} \beta$ synthesis in nonacinar 

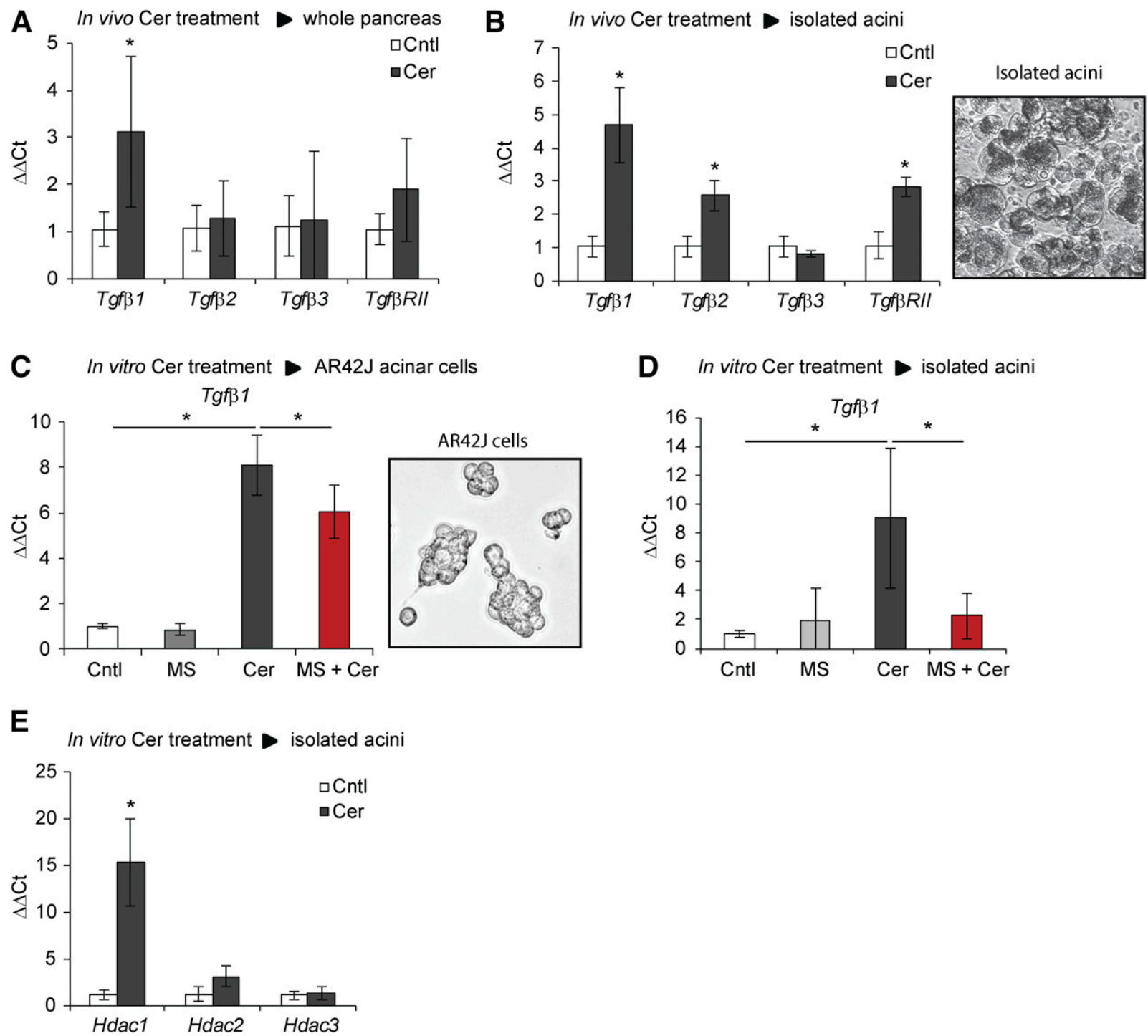

Fig. 5. MS-275 administration inhibits TGF $\beta$ expression in isolated acinar cells. (A) qPCR of TGF $\beta$ isoforms and TGF $\beta$ receptor 2 (TGF $\beta$ R2) expression in the whole pancreas 24 hours after cerulein treatment. (B) qPCR of TGF $\beta$ isoforms and TGF $\beta$ receptor 2 (TGF $\beta$ R2) expression in primary acini isolated 24 hours after in vivo cerulein treatment. Right panel: Micrograph of isolated pancreatic acini. (C) qPCR of TGF $\beta 1$ expression in AR42J acinar cells upon cerulein stimulation. Right panel: Micrograph of AR42J cells. (D) qPCR of TGF $\beta 1$ expression in isolated acinar cells upon in vitro treatment with cerulein in the presence or absence of MS-275. (E) qPCR of class 1 HDAC expression in isolated acinar cells treated in vitro with cerulein. Results are average \pm S.D. $(n=5$ to 6$),{ }^{*} P<0.05$

cells may also depend on HDAC activity. In fact, treatment of isolated PSCs with the pan-HDAC inhibitor sodium valproate inhibits TGF $\beta$ expression and collagen synthesis in these cells (Bülow et al., 2007). Furthermore, another possible source of TGF $\beta$ production is inflammatory cells, which are recruited to the pancreas during the development of pancreatitis. This is particularly interesting as we recently demonstrated that MS-275 treatment effectively reduced the levels of inflammation during the course of acute and chronic pancreatitis (Bombardo et al., 2017). Crosstalk between PSCs and distinct leukocyte populations, including macrophages, promotes PSC activation and fibrosis during chronic pancreatitis (Xue et al., 2015). Future studies using coculture of acinar cells, PSC, and leukocytes are warranted to dissect the contribution of the individual cell types in the production of $\mathrm{TGF} \beta$ upon treatment with MS-275 and the effect on PSC activation.
Although it is possible that reduced inflammation upon MS-275 administration leads to reduced fibrosis during chronic pancreatitis, a recent study revealed that development of inflammation and fibrosis is two independent and, accordingly, not causal events in this disease. Specifically, using transgenic mice deficient in Cxcr2, the authors observed almost complete ablation of inflammatory cell infiltration upon chronic pancreatitis; however, this limited inflammatory reaction did not prevent PSC activation; consequently, fibrosis levels were comparable in transgenic and wild-type control mice (Steele et al., 2015). This striking example implies that signaling molecules derived from inflammatory cells may play a minor role in the development of pancreatic fibrosis during chronic pancreatitis.

Collectively, our results integrate with the current body of evidence demonstrating the crucial role of HDACs in the development of fibrotic diseases. In this regard, compelling 
evidence demonstrates that HDAC activity is necessary for activation of hepatic stellate cells in vitro (reviewed in Chen et al., 2015). The requirement of HDAC activity in driving myofibroblastic differentiation and ECM protein synthesis was also observed in different fibroblast populations present in skin, lung, and kidney (Glenisson et al., 2007; Yoshikawa et al., 2007; Guo et al., 2009). Moreover, the use of different HDAC inhibitors showed beneficial effects in the treatment of hepatic, renal, cardiac, and pulmonary fibrosis in vivo (Kee et al., 2013; Liu et al., 2013; Van Beneden et al., 2013; Khan and Jena, 2014; Nural-Guvener et al., 2014; Chen et al., 2015; Choi et al., 2015; Korfei et al., 2015). This finding suggests that epigenetic mechanisms controlled by HDACs may be conserved in the development of different fibrotic diseases.

Conclusion. Counteracting the development of pancreatic fibrosis is a major and elusive therapeutic goal in the context of chronic pancreatitis. Our data revealed a potent antifibrotic effect of MS-275 treatment, which is mediated at least in part by suppression of TGF $\beta$ expression in acinar cells; however, it is possible that downregulation of additional factors contributes to the observed phenotype. In this context, it is worth mentioning that pancreatic expression of interleukins-1 and -6 , interleukins known to promote autocrine and paracrine activation of PSC (Bynigeri et al., 2017), was reduced upon MS-275 treatment (Bombardo et al., 2017).

Collectively, our data suggest on one hand that class $1 \mathrm{HDAC}$ activity is critical for the timely controlled epigenetic regulation of key signaling molecules driving the development of fibrosis in this organ. On the other hand, our data provide a new perspective on the cell types involved in regulating the process and highlights the possibility that acinar cells act as active mediators of pancreatic fibrosis.

These results harbor important implications to explore further the therapeutic potential of MS-275 in the context of chronic pancreatitis patients. Additional studies that include experimental models with increased severity of pancreatitis and autoimmune pancreatitis are warranted to define the effect of MS-275 in a broader spectrum of disease manifestations. Moreover, investigations using conditional knockout mouse models are needed to achieve a global understanding of the individual HDAC isoforms' functions in the different cell types that are involved in the development of this disease.

\section{Acknowledgments}

We thank Theresia Reding and Udo Ungetuem for invaluable support and technical assistance.

\section{Authorship Contributions}

Participated in research design: Bombardo, Graf, Sonda.

Conducted experiments: Bombardo, Chen, Malagola, Saponara.

Performed data analysis: Bombardo, Chen, Malagola, Saponara.

Wrote or contributed to the writing of the manuscript: Bombardo, Hills, Graf, Sonda.

\section{References}

Algül H, Wagner M, Lesina M, and Schmid RM (2007) Overexpression of ErbB2 in the exocrine pancreas induces an inflammatory response but not increased proliferation. Int $J$ Cancer 121:1410-1416.

Apte M, Pirola R, and Wilson J (2011) The fibrosis of chronic pancreatitis: new insights into the role of pancreatic stellate cells. Antioxid Redox Signal 15 2711-2722.

Bombardo M, Saponara E, Malagola E, Chen R, Seleznik GM, Haumaitre C, Quilichini E, Zabel A, Reding T, Graf R, et al. (2017) Class I histone deacetylase inhibition improves pancreatitis outcome by limiting leukocyte recruitment and acinar-to-ductal metaplasia. Br J Pharmacol 174:3865-3880.

Bülow R, Fitzner B, Sparmann G, Emmrich J, Liebe S, and Jaster R (2007) Antifibrogenic effects of histone deacetylase inhibitors on pancreatic stellate cells. Biochem Pharmacol 74:1747-1757.

Bynigeri RR, Jakkampudi A, Jangala R, Subramanyam C, Sasikala M, Rao GV Reddy DN, and Talukdar R (2017) Pancreatic stellate cell: Pandora's box for pancreatic disease biology. World J Gastroenterol 23:382-405.

Chen PJ, Huang C, Meng XM, and Li J (2015) Epigenetic modifications by histone deacetylases: biological implications and therapeutic potential in liver fibrosis. Biochimie 116:61-69.

Choi SY, Ryu Y, Kee HJ, Cho SN, Kim GR, Cho JY, Kim HS, Kim IK, and Jeong $\mathrm{MH}$ (2015) Tubastatin A suppresses renal fibrosis via regulation of epigenetic histone modification and Smad3-dependent fibrotic genes. Vascul Pharmacol 72:130-140.

Dalgard CL, Van Quill KR, and O'Brien JM (2008) Evaluation of the in vitro and in vivo antitumor activity of histone deacetylase inhibitors for the therapy of retinoblastoma. Clin Cancer Res 14:3113-3123.

DiMagno EP and DiMagno MJ (2016) Chronic pancreatitis: landmark papers, management decisions, and future. Pancreas 45:641-650.

DiMagno MJ and DiMagno EP (2013) Chronic pancreatitis. Curr Opin Gastroenterol 29:531-536.

Glenisson W, Castronovo V, and Waltregny D (2007) Histone deacetylase 4 is required for TGFbeta1-induced myofibroblastic differentiation. Biochim Biophys Acta 1773:1572-1582.

Graf R, Schiesser M, Lüssi A, Went P, Scheele GA, and Bimmler D (2002) Coordinate regulation of secretory stress proteins (PSP/reg, PAP I, PAP II, and PAP III) in the rat exocrine pancreas during experimental acute pancreatitis. J Surg Res 105 136-144.

Guo W, Shan B, Klingsberg RC, Qin X, and Lasky JA (2009) Abrogation of TGFbeta1-induced fibroblast-myofibroblast differentiation by histone deacetylase inhibition. Am J Physiol Lung Cell Mol Physiol 297:L864-L870.

He J, Sun X, Qian KQ, Liu X, Wang Z, and Chen Y (2009) Protection of ceruleininduced pancreatic fibrosis by pancreas-specific expression of Smad7. Biochim Biophys Acta 1792:56-60.

Kee HJ, Bae EH, Park S, Lee KE, Suh SH, Kim SW, and Jeong MH (2013) HDAC inhibition suppresses cardiac hypertrophy and fibrosis in DOCA-salt hypertensive rats via regulation of HDAC6/HDAC8 enzyme activity. Kidney Blood Press Res 37: $229-239$.

Khan S and Jena G (2014) Sodium butyrate, a HDAC inhibitor ameliorates eNOS, iNOS and TGF- $\beta 1$-induced fibrogenesis, apoptosis and DNA damage in the kidney of juvenile diabetic rats. Food Chem Toxicol 73:127-139.

Korfei M, Skwarna S, Henneke I, MacKenzie B, Klymenko O, Saito S, Ruppert C, von der Beck D, Mahavadi P, Klepetko W, et al. (2015) Aberrant expression and activity of histone deacetylases in sporadic idiopathic pulmonary fibrosis. Thorax $\mathbf{7 0}$ : 1022-1032.

Kruse ML, Hildebrand PB, Timke C, Fölsch UR, and Schmidt WE (2000) TGFbeta1 autocrine growth control in isolated pancreatic fibroblastoid cells/stellate cells in vitro. Regul Pept 90:47-52.

Li X, Nania S, Fejzibegovic N, Moro CF, Klopp-Schulze L, Verbeke C, Löhr JM, and Heuchel RL (2016) Cerulein-induced pancreatic fibrosis is modulated by Smad7, the major negative regulator of transforming growth factor- $\beta$ signaling. Biochim Biophys Acta 1862:1839-1846.

Liu N, He S, Ma L, Ponnusamy M, Tang J, Tolbert E, Bayliss G, Zhao TC, Yan H, and Zhuang S (2013) Blocking the class I histone deacetylase ameliorates renal fibrosis and inhibits renal fibroblast activation via modulating TGF-beta and EGFR signaling. PLoS One 8:e54001.

McDonnell F, O'Brien C, and Wallace D (2014) The role of epigenetics in the fibrotic processes associated with glaucoma. J Ophthalmol 2014:750459.

Menke A, Yamaguchi H, Gress TM, and Adler G (1997) Extracellular matrix is reduced by inhibition of transforming growth factor beta1 in pancreatitis in the rat. Gastroenterology 113:295-303.

Moran-Salvador E and Mann J (2017) Epigenetics and liver fibrosis. Cell Mol Gastroenterol Hepatol 4:125-134.

Muller-Pillasch F, Menke A, Yamaguchi H, Elsasser HP, Bachem M, Adler G, and Gress TM (1999) TGFbeta and the extracellular matrix in pancreatitis. Hepatogastroenterology 46:2751-2756.

Murphy SP, Lee RJ, McClean ME, Pemberton HE, Uo T, Morrison RS, Bastian C, and Baltan S (2014) MS-275, a class I histone deacetylase inhibitor, protects the p53-deficient mouse against ischemic injury. $J$ Neurochem 129 : $509-515$.

Nguyên TL, Abdelbary H, Arguello M, Breitbach C, Leveille S, Diallo JS, Yasmeen A, Bismar TA, Kirn D, Falls T, et al. (2008) Chemical targeting of the innate antiviral response by histone deacetylase inhibitors renders refractory cancers sensitive to viral oncolysis. Proc Natl Acad Sci USA 105:14981-14986.

Nural-Guvener HF, Zakharova L, Nimlos J, Popovic S, Mastroeni D, and Gaballa MA (2014) HDAC class I inhibitor, Mocetinostat, reverses cardiac fibrosis in heart failure and diminishes CD90 + cardiac myofibroblast activation. Fibrogenesis Tissue Repair 7:10.

Pang M and Zhuang S (2010) Histone deacetylase: a potential therapeutic target for fibrotic disorders. J Pharmacol Exp Ther 335:266-272.

Royce SG, Moodley Y, and Samuel CS (2014) Novel therapeutic strategies for lung disorders associated with airway remodelling and fibrosis. Pharmacol Ther 141: $250-260$.

Schuetze KB, Koch KA, and McKinsey TA (2016) The potential of targeting epigenetic regulators for the treatment of fibrotic cardiac diseases. Future Med Chem 8 : 1533-1536.

Silva A, Weber A, Bain M, Reding T, Heikenwalder M, Sonda S, and Graf R (2011) COX-2 is not required for the development of murine chronic pancreatitis. Am $J$ Physiol Gastrointest Liver Physiol 300:G968-G975. 
Steele CW, Karim SA, Foth M, Rishi L, Leach JD, Porter RJ, Nixon C, Jeffry Evans TR, Carter CR, Nibbs RJ, et al. (2015) CXCR2 inhibition suppresses acute and chronic pancreatic inflammation. J Pathol 237:85-97.

Van Beneden K, Mannaerts I, Pauwels M, Van den Branden C, and van Grunsven LA (2013) HDAC inhibitors in experimental liver and kidney fibrosis. Fibrogenesis Tissue Repair 6:1.

Weigel C, Schmezer P, Plass C, and Popanda O (2015) Epigenetics in radiationinduced fibrosis. Oncogene 34:2145-2155.

Xue J, Sharma V, Hsieh MH, Chawla A, Murali R, Pandol SJ, and Habtezion A (2015) Alternatively activated macrophages promote pancreatic fibrosis in chronic pancreatitis. Nat Commun 6:7158.

Yang IV and Schwartz DA (2015) Epigenetics of idiopathic pulmonary fibrosis. Transl Res 165:48-60.
Yoo BM, Yeo M, Oh TY, Choi JH, Kim WW, Kim JH, Cho SW, Kim SJ, and Hahm KB (2005) Amelioration of pancreatic fibrosis in mice with defective TGF-beta signaling. Pancreas 30:e71-e79.

Yoshikawa M, Hishikawa K, Marumo T, and Fujita T (2007) Inhibition of histone deacetylase activity suppresses epithelial-to-mesenchymal transition induced by TGF-beta1 in human renal epithelial cells. J Am Soc Nephrol 18:58-65.

Address correspondence to: Sabrina Sonda, Pancreatitis Research Laboratory, Department of Visceral and Transplantation Surgery, University Hospital Zurich, Rämistrasse 100, 8091 Zurich, Switzerland. E-mail: sabrina. sonda@usz.ch 